ESJ Humanities

\title{
Ville et Evenement : Analyse de l'inscription Territoriale de la « Fete du 15 Aout » Dans la Ville de Savalou (Centre Benin)
}

\author{
Vissoh Ahotondji Sylvain
}

Laboratoire d'Etudes des Dynamiques Urbaines et Régionales (LEDUR),

Département de Géographie et Aménagement du Territoire (DGAT),

Université d'Abomey-Calavi, Benin

Doi:10.19044/esj.2021.v17n42p69

Submitted: 11 September 2021

Accepted: 25 December 2021

Published: 31 December 2021
Copyright 2021 Author(s)

Under Creative Commons BY-NC-ND

4.0 OPEN ACCESS

Cite As:

Sylvain V.A. (2021). Ville et Evenement: Analyse de l'inscription Territoriale de la "Fete du 15 Aout » Dans la Ville de Savalou (Centre Benin). European Scientific Journal, ESJ, 17 (42), 69. https://doi.org/10.19044/esj.2021.v17n42p69

\section{Résumé}

Les villes accueillent souvent des évènements qui, de par leur nature et les manifestations qu'ils engendrent, marquent la trame urbaine et contribuent au développement local. Le but de la présente recherche est d'analyser l'inscription territoriale de la « fête du 15 août », le plus important évènement qu'accueille, chaque année, la ville de Savalou. L'approche méthodologique adoptée combine les recherches documentaires notamment sur la thématique ville et évènement, les enquêtes de terrain à travers l'observation participante des manifestations des éditions 2019 et 2021 de la fête du 15 août, les entretiens semi directifs avec les autorités communales et les questionnaires avec les promoteurs de réceptifs hôteliers, de restaurants ainsi que quelques forains et visiteurs urbains. Au total, 173 personnes ont été interrogées dans le cadre de cette recherche. Ces personnes ont été retenues par la méthode de choix raisonné. Les méthodes d'analyse de contenu et de statistiques descriptives ont permis de traiter les données et informations recueillies et le modèle SWOT ou FFOM (Forces, Faiblesses, Opportunités et Menaces) a été utilisé pour analyser les résultats obtenus. Il ressort des analyses que cinq (05) manifestations clés sont organisées dans le cadre de la « fête du 15 août »: il s'agit du rituel d'autorisation de la consommation des nouvelles ignames, la Grande Foire Artisanale de Savalou (GFAS), l'élection de Miss Savalou (la plus belle fille de la Commune de Savalou), la finale de la coupe de football de la commune et les concerts. Au plan spatial, 
l'évènement se déroule en plusieurs lieux à savoir le palais royal, la paroisse de l'église catholique, la maison des jeunes, le stade omnisport de la ville, la place Soha et le site de la foire. Il n'existe pas une structure officielle qui assure le portage de l'évènement; chaque manifestation est organisée par un acteur donné sans qu'aucune coordination ne soit mise en place. Par ailleurs, très peu de recettes sont collectées par la mairie à l'occasion de cet évènement. On en déduit que l'évènement profite financièrement plus aux acteurs privés qu'à la municipalité.

Mots-clés : Ville de Savalou, évènement urbain, fête identitaire, développement local

\title{
City and Event: Analysis of the Territorial Registration of the "Feast of August 15 " in the City of Savalou (Center Benin)
}

\author{
Vissoh Ahotondji Sylvain \\ Laboratoire d'Etudes des Dynamiques Urbaines et Régionales (LEDUR), \\ Département de Géographie et Aménagement du Territoire (DGAT), \\ Université d'Abomey-Calavi, Benin
}

\begin{abstract}
Cities often host events which, by their nature and the events they generate, mark the urban fabric and contribute to local development. The aim of this research is to analyze the territorial inscription of the "feast of August 15 ", the most important event hosted each year by the city of Savalou. The methodological approach adopted combines documentary research, in particular on the city and event theme, field surveys through the participant observation of the events of the 2019 and 2021 editions of the August 15 festival, semi-structured interviews with the municipal authorities and questionnaires with the promoters of hotel receptives, restaurants as well as a few fairgrounds and urban visitors. A total of 173 people were interviewed for this research. These people were selected by the reasoned choice method. The methods of content analysis and descriptive statistics were used to process the data and information collected and the SWOT or SWOT model (Strengths, Weaknesses, Opportunities and Threats) was used to analyze the results obtained. It emerges from the analyzes that five (05) key events are organized within the framework of the "feast of August 15": it is about the ritual of authorization of the consumption of new yams, the Great Artisanal Fair of Savalou (GFAS), the election of Miss Savalou (the most beautiful girl in the Municipality of Savalou), the final of the municipal football cup and the concerts. Spatially, the event takes place in several locations, namely the
\end{abstract}


Royal Palace, the parish of the Catholic Church, the youth center, the city's sports stadium, Soha Square and the site of the fair. There is no official structure that ensures the portering of the event; each event is organized by a given actor without any coordination being put in place. In addition, very little revenue is collected by the town hall during this event. We deduce that the event financially benefits private actors more than the municipality.

Keywords: City of Savalou, urban event, identity celebration, local development

\section{Introduction}

La ville de Savalou accueille chaque année un grand évènement festif et culturel qui rythme le calendrier des ressortissants de la commune. Dénommée fête de l'igname ou fête du 15 août, cet évènement est à la fois une fête patronale, religieuse et identitaire des filles et fils de la Commune de Savalou. Il se déroule du 14 au 16 août de chaque année avec comme point d'orgue des manifestations, la journée du 15 août.

D'après Gagnebien (2011), les évènements qui se déroulent en ville, constituent des facteurs incontournables dans la compréhension et l'analyse des faits économiques, sociaux et politiques urbains. Et pour Miranda (2010), les événements urbains festifs s'imposent non seulement comme de nouvelles pratiques sociales, mais aussi en tant qu'action urbaine et objet d'analyse.

Qu'ils soient festifs ou culturels, les évènements urbains relèvent de la nécessité, pour un groupe social de se réaffirmer en décidant d'un temps, d'un espace et d'une activité à la fois très codifiée et décalée par rapport à la vie ordinaire (Barthon et al., 2007).

A l'heure de la décentralisation, la mobilisation des ressources financières locales est un véritable défi pour l'ensemble des collectivités territoriales décentralisées (Nkwenka Nyanda et al., 2020) et les évènements urbains sont souvent des occasions de mobilisation des investisseurs locaux, nationaux voire internationaux autour d'une vision assortie de projets de développement. Par ailleurs, la médiatisation de ces évènements permet d'améliorer l'image des territoires qui les accueillent; ce qui justifie les compétitions de plus en plus fortes entre territoires pour abriter des évènements de grande notoriété (Lallement, 2007). Pour Nassourou et Sani (2019), les autorités locales doivent créer les conditions d'un développement durable en tirant partie de tous les évènements qui se déroulent sur leur territoire.

La fête du 15 août étant le plus grand évènement qu'abrite la ville de Savalou, il est intéressant d'analyser son inscription territoriale à travers les questionnements ci-après : 
Que représente la fête de 15 août pour les autorités locales ? Quelle stratégie développent-elles pour son organisation? Quelles sont les marques de cet évènement dans l'espace urbain ? Quelles sont les retombées de cet évènement pour la mairie et les autres acteurs impliqués dans son organisation ? La présente recherche se propose de répondre à ces questionnements en analysant l'inscription territoriale de cette fête.

\section{Matériels et méthodes}

\subsection{Cadrage géographique de l'étude}

La ville de Savalou, objet de la présente recherche, est située dans le Département des Collines. Chef-lieu de la commune du même nom, elle est comprise entre $7^{\circ} 56^{\prime}$ et $8^{\circ}$ de latitude Nord et $1^{\circ} 59^{\prime}$ et $2^{\circ}$ de longitude Est ; la ville est limitée au Nord par les Arrondissements de Ouèssè et d'Attakè, au Sud par celui de Djalloukou, à l'Est par les Arrondissements de Logozohê et de Gobada et à l'Ouest par ceux de Tchetti et de Doumè (figure 1). Les populations de Savalou s'adonnent à plusieurs activités: l'agriculture (production de céréales, légumineuses, tubercules, produits maraîchers), la transformation des produits agricoles (transformation du manioc en gari, de l'arachide en galette), l'élevage (volaille et petits ruminants), le commerce (achat et vente de produits agricoles et manufacturés), l'artisanat (couture, coiffure, etc.) et les services (restauration, transport). 


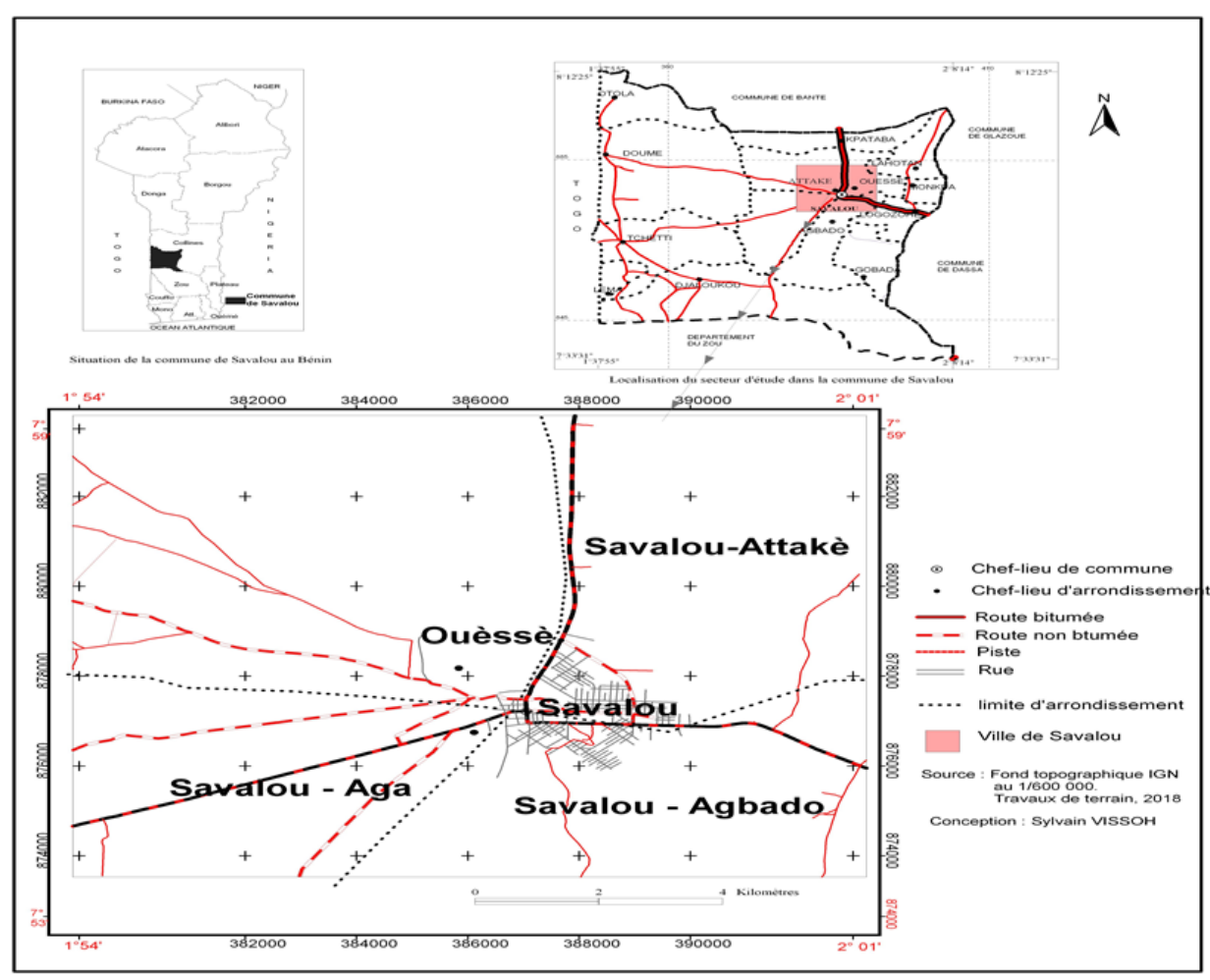

Figure 1 : Situation géographique et administrative de la ville de Savalou

\subsection{Nature des données collectées}

Les données collectées portent sur le profil socio-professionnel des visiteurs urbains de la fête du 15 août, la nature des spectacles déroulés, l'effectif des forains, la nature des produits échangés au cours de la foire du 15 août, les types de taxes collectées par l'administration communale et leurs montants respectifs.

\subsection{Techniques, outils et matériels de collecte des données}

La collecte des données a démarré par la documentation; celle-ci a permis de comprendre la place des évènements dans le développement des villes, d'une part, et d'identifier les différentes manifestations organisées à l'occasion de la fête du 15 août ainsi que les acteurs impliqués, d'autre part. Une observation participante a été effectuée sur deux éditions de la fête, celles de 2019 et de 2021(pour des raisons liées à la pandémie du COVID 19, l'édition 2020 de la fête a été annulée). Les observations ont été faites du 14 au 16 août de chaque année (2019 et 2021). Des enquêtes ont été effectuées auprès des principaux acteurs de l'organisation de l'évènement à savoir les autorités et agents communaux, les promoteurs d'hôtels et de restaurants, les 
visiteurs urbains de la période couverte par la fête, les promoteurs de la foire et quelques forains. A cet effet, des entretiens semi directifs ont été organisés avec le maire, deux (02) chefs d'arrondissement urbains et quatre (04) agents communaux. Par ailleurs, des questionnaires ont été adressés à cinq (05) promoteurs d'hôtels, treize (13) propriétaires de restaurant et vingt-cinq (25) gérants de buvettes. Ces promoteurs et propriétaires ont été choisis en tenant compte de la taille de leur entreprise. Des questionnaires ont été également adressés à cent (100) visiteurs urbains et vingt (20) forains, choisis au hasard pour apprécier leurs motivations et attentes de la fête du 15 août. Enfin des interviews ont eu lieu avec le promoteur de Miss Savalou et deux responsables de l'organisation de la finale de la coupe de football de Savalou et de spectacle de musique à travers des questionnaires. Au total, 173 personnes ont été interrogées dans le cadre de cette recherche. Les outils utilisés sont la grille d'observation (qui nous a permis d'être attentif à l'occupation des lieux de spectacles, l'ambiance au cours des spectacles, les tranches d'âge selon les spectacles, la nature des produits exposés aux abords des rues et sur le site de la foire), le guide d'entretien adressé aux autorités locales et agents communaux, et le questionnaire utilisé pour le reste des acteurs interrogés. Un appareil photographique numérique et un GPS ont été utilisés pour, respectivement, prendre des photos et enregistrer les coordonnées géographiques des lieux des manifestations.

\subsection{Méthodes de traitement des données collectées et analyse des résultats}

Au terme des travaux de terrain, les fiches d'enquête ont été regroupées par catégorie d'acteurs et il a été procédé à un dépouillement manuel. Une analyse de contenu a été effectuée sur les données qualitatives et un traitement statistique a été fait sur les données quantitatives à travers le calcul de fréquence selon la formule $\mathrm{F}=\left(\mathrm{n}_{\mathrm{i}} / \mathrm{N}\right) \times 100$ où $\mathrm{F}$ désigne la fréquence, $\mathrm{n}_{\mathrm{i}}$ est le nombre de personnes ayant fourni la réponse i et $\mathrm{N}$ le nombre total de personnes interrogées. Les résultats issus de ce traitement ont été analysés suivant le modèle SWOT ou FFOM (Forces, Faiblesses, Opportunités et Menaces).

\section{Résultats}

\subsection{Origine de la fête du 15 août}

D'après les informations reçues sur le terrain, l'origine de la fête de l'igname ou fête du 15 août remonterait au XVII ${ }^{\text {ème }}$ siècle (Mairie de Savalou, 2009). En effet, le roi Soha, Gbaguidi $1^{\text {er }}$ (fondateur et premier roi de Tchébélou), ayant très tôt constaté l'aptitude des sols de son royaume à la culture de l'igname, s'y est investi et a encouragé ses sujets à faire de même. L'igname fût donc l'une des plus importantes cultures pratiquées dans le 
royaume. Pour célébrer les succès enregistrés dans la production de cette spéculation, il fut décidé de l'organisation d'une cérémonie au début de chaque récolte. Cette cérémonie serait l'occasion de remercier les dieux pour leur soutien et également de les implorer pour la saison suivante. Au cours de cette cérémonie, qui se tient autour du 10 août (milieu ou fin de la deuxième semaine du mois d'août), l'igname est préparée et offerte aux divinités du royaume. Ce n'est qu'après cette offrande que les filles et fils du royaume sont autorisés à consommer les nouvelles ignames. C'était une cérémonie non seulement très attendue dans le royaume mais aussi très mobilisatrice de monde. Avec l'arrivée du catholicisme dans le royaume, une paroisse a été construite à laquelle fut donnée le nom « Paroisse Notre Dame d'Assomption de Savalou ». La fête de l'Assomption de l'Eglise Catholique, qui se célèbre les 15 août, devient donc fête patronale de cette paroisse. La date de sa célébration (15 août) se trouve être assez rapprochée de celle de la fête de l'igname. Le palais royal et les responsables de la paroisse ont donc décidé de faire coïncider les deux fêtes pour en faire un évènement plus grandiose. Ce rapprochement entre le palais royal et l'église catholique a favorisé l'ancrage de la religion catholique au sein de la population en même temps qu'elle conféra plus d'éclat à la célébration de la fête de l'igname. Le rituel de la fête de l'igname se déroule très tôt le matin pour permettre à ceux qui le désirent de se rendre à la messe, souvent autour de 10 heures ( $9 \mathrm{~h}$ en temps universel) et les réjouissances populaires (danses, jeux divers, etc.) se poursuivent dans l'après-midi. Deux fêtes sont donc célébrées le même jour mais pour des raisons différentes : pour les chrétiens, la fête du 15 août marque la montée au ciel du corps et de l'âme de la Vierge Marie, mère de Jésus et pour les non chrétiens, la fête du 15 août est destinée à remercier les divinités protectrices de la production agricole, en particulier celle de l'igname. Le rapprochement entre les deux communautés (chrétienne et non chrétienne) est donc lié à la date et non au contenu et signification de la fête.

La photo 1 présente une séquence des manifestations qui se déroulent au niveau du palais royal 


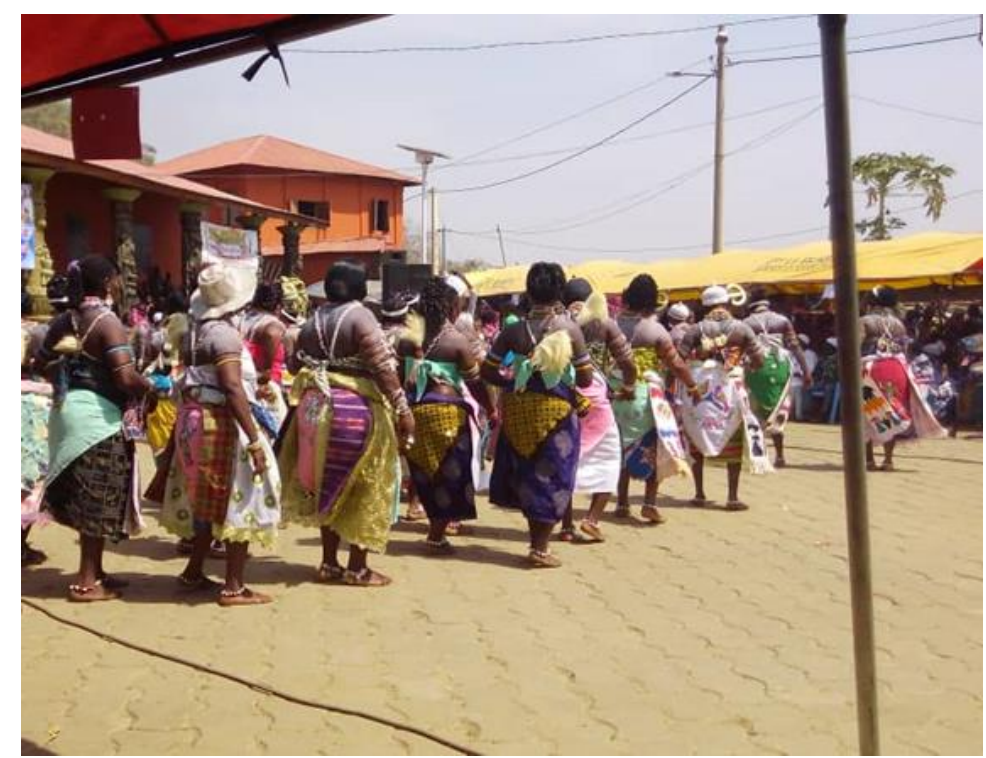

Photo 1 : Vue partielle des manifestations du 15 août au palais royal

Prise de vue : Vissoh, août 2019

\subsection{Acteurs impliqués et rôles dans l'organisation de l'évènement}

Quatre catégories d'acteurs interviennent dans l'organisation et la tenue de la fête du 15 août : le palais royal, la Mairie, l'Eglise catholique et les opérateurs économiques.

$\mathrm{Au}$ départ, le palais royal s'investissait beaucoup plus dans l'aspect cultuel et culturel de la fête. Mais au fil du temps, son rôle a évolué au point où il se positionne aujourd'hui comme l'un des acteurs clés de la mobilisation de ressources au profit de l'évènement. Cela a été particulièrement manifeste au cours du règne du roi Tossoh Gbaguidi (XIII ${ }^{\text {ème }}$ roi de Savalou). Ce dernier, grâce à son charisme, sa notoriété et ses relations, a su mobiliser, non seulement les filles et fils de la commune mais aussi d'autres personnalités, ressortissant des autres régions du Bénin autour de l'évènement. C'est ainsi que la ville de Savalou est souvent prise d'assaut par les hommes politiques du pays surtout si la fête se déroule à la veille d'échéances électorales. On peut citer les éditions 1995, 2000, 2005, 2010 et 2015 de la fête du 15 août où l'on était alors à la veille des élections présidentielles de 1996, 2001, 2006, 2011 et 2016.

La Mairie est beaucoup plus dans le rôle administratif de l'organisation. Elle travaille à créer les meilleures conditions aux participants à la fête notamment les opérateurs économiques. Ceci se traduit par exemple par la mise à disposition d'un site dédié à l'organisation d'une foire agricole et artisanale à l'occasion de la fête.

La paroisse de l'église catholique est incontestablement l'un des acteurs clés de l'organisation et de la réussite de la fête. En effet, il n'y a pas 
de fête de 15 août sans une grande messe à la paroisse catholique Notre Dame d'Assomption de Savalou. Cette messe est l'une des grandes attractions de la fête. Le clou du déroulement de cette messe est la séquence relative à l'action de grâces au cours de laquelle les invités rivalisent de générosité pour faire des dons en nature (matériaux de construction) mais aussi financiers. Ces dons permettent de soutenir les responsables de la paroisse dans leurs œuvres caritatives (dons à des orphelinats, soutien à la construction de stations paroissiales dans les villages, etc.). A chaque célébration de la fête, la question qui se pose est de savoir combien de millions seront mobilisés et si le montant de l'année pourra atteindre ou dépasser celui de l'année antérieure ? La fête du 15 août 2005, veille des élections présidentielles devant marquer la fin des deux mandats constitutionnels (démocratiques) du Général Mathieu KEREKOU, a été l'une des plus fructueuses pour la paroisse. Au cours de cette édition de 2005, environ vingt (20) millions de francs CFA auraient été mobilisés cette année-là.

Les opérateurs économiques sont de loin ceux qui animent la fête de par leur nombre et les diverses activités qu'ils proposent. Ces activités portent pour, l'essentiel, sur l'organisation de manifestations (concours de beauté, miss 15 août, prestations d'artistes modernes et traditionnels, match de football, etc.) et la vente de produits agricoles (surtout les ignames de la nouvelle saison) et artisanaux (artisans locaux mais aussi nationaux voire internationaux (Togolais et Burkinabè). Pour permettre à ces opérateurs d'exercer leurs activités dans un cadre plus organisé, la mairie a initié à partir de 2002, une foire agro-artisanale à l'occasion de la fête du 15 août. La première édition de cette foire (août 2002) s'est déroulée sur un site attenant au carrefour dit « rond-point du CARDER », l'un des lieux stratégiques de la ville. Au fil des années, ce site s'est révélé trop exigu pour contenir les forains de plus en plus nombreux. Un autre site a été alors identifié au quartier Ahossèdo, non loin de la gare des bus et camions gros porteurs de la ville (photo 2). 


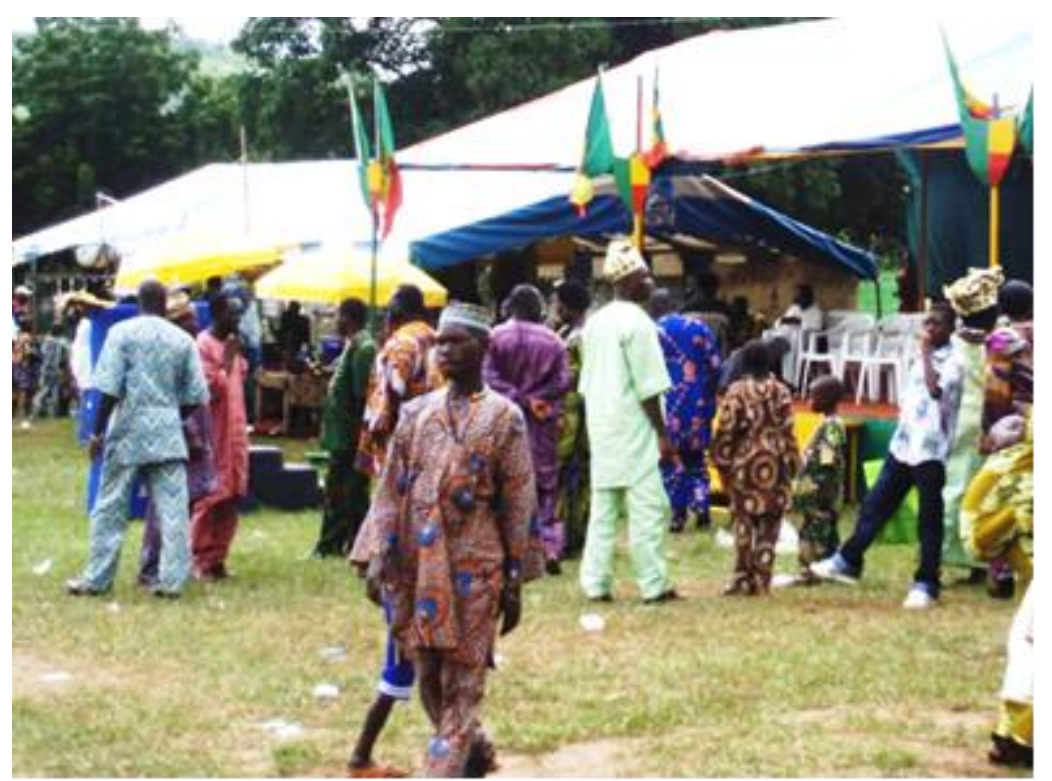

Photo 2 : Vue partielle des participants à la foire du 15 août 2021, au quartier Ahossèdo Prise de vue : Vissoh, août 2021

La photo 2 montre une vue partielle des participants à la foire agricole et artisanale du 15 août 2021. On peut observer sur la photo que toutes les catégories sociales (hommes, femmes, jeunes) visitent cette foire à la découverte des produits agricoles et artisanaux de la commune mais aussi des autres contrées voire pays dont les représentants participent à la foire.

Cette activité comme les autres ci-dessus évoquées laissent des marques dans l'espace urbain.

\subsection{Les marques de la fête du 15 août dans l'espace urbain}

Sur le plan spatial, les marques de la fête se sont d'abord fait observer au niveau du palais royal, ensuite au niveau de la paroisse de l'église catholique pour s'étendre progressivement à d'autres endroits de la ville. La figure 2 suivante permet de voir les lieux de la fête dans l'espace urbain. 


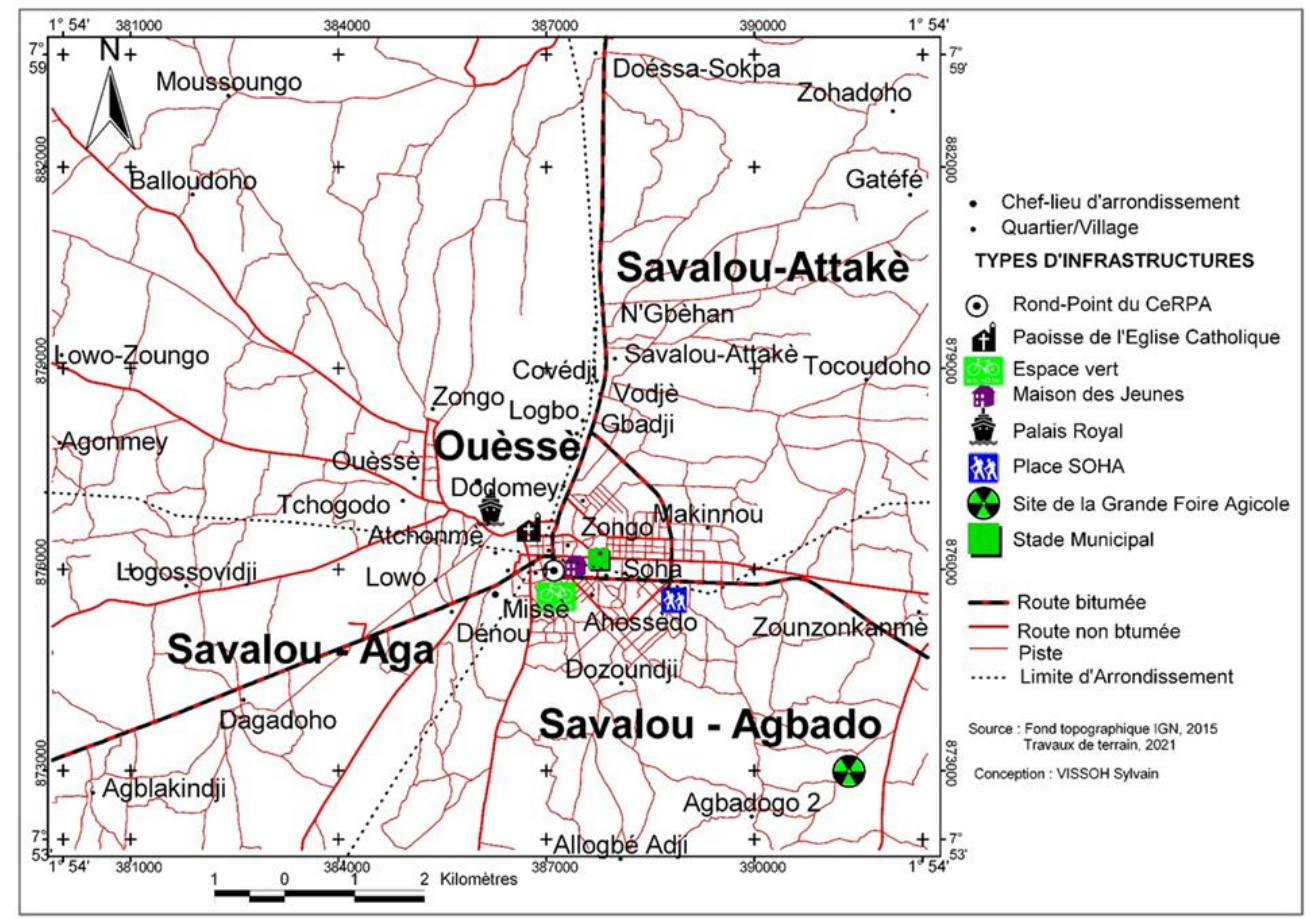

Figure 2 : Lieux de la fête du 15 août dans la ville de Savalou

Source : Résultats d'enquêtes, août 2021

Il ressort de cette figure que tous les lieux stratégiques de la ville à savoir le Rond-point du CeRPA, la Paroisse de l'Eglise Catholique, la maison des jeunes, le Palais Royal, le stade municipal sont aujourd'hui pris d'assaut par les manifestations de la fête. En dehors du site de la foire agricole et artisanale qui est spécifiquement aménagé pour la fête du 15 août, les autres infrastructures et équipements sont construits indépendamment de la fête ; mais leurs fonctions dans la ville sont aujourd'hui rehaussées par les activités qu'ils abritent à l'occasion de cette fête. La ville de Savalou vit désormais au rythme de cette fête. Tout autre évènement, de quelque nature que ce soit, se décide et s'organise en référence à cette fête.

\subsection{Motivations des visiteurs de la ville de Savalou, à l'occasion de la fête du 15 août}

A l'occasion de la fête du 15 août, les personnes qui visitent la ville de Savalou le font pour plusieurs raisons comme le montre la figure 3 ci-après : 


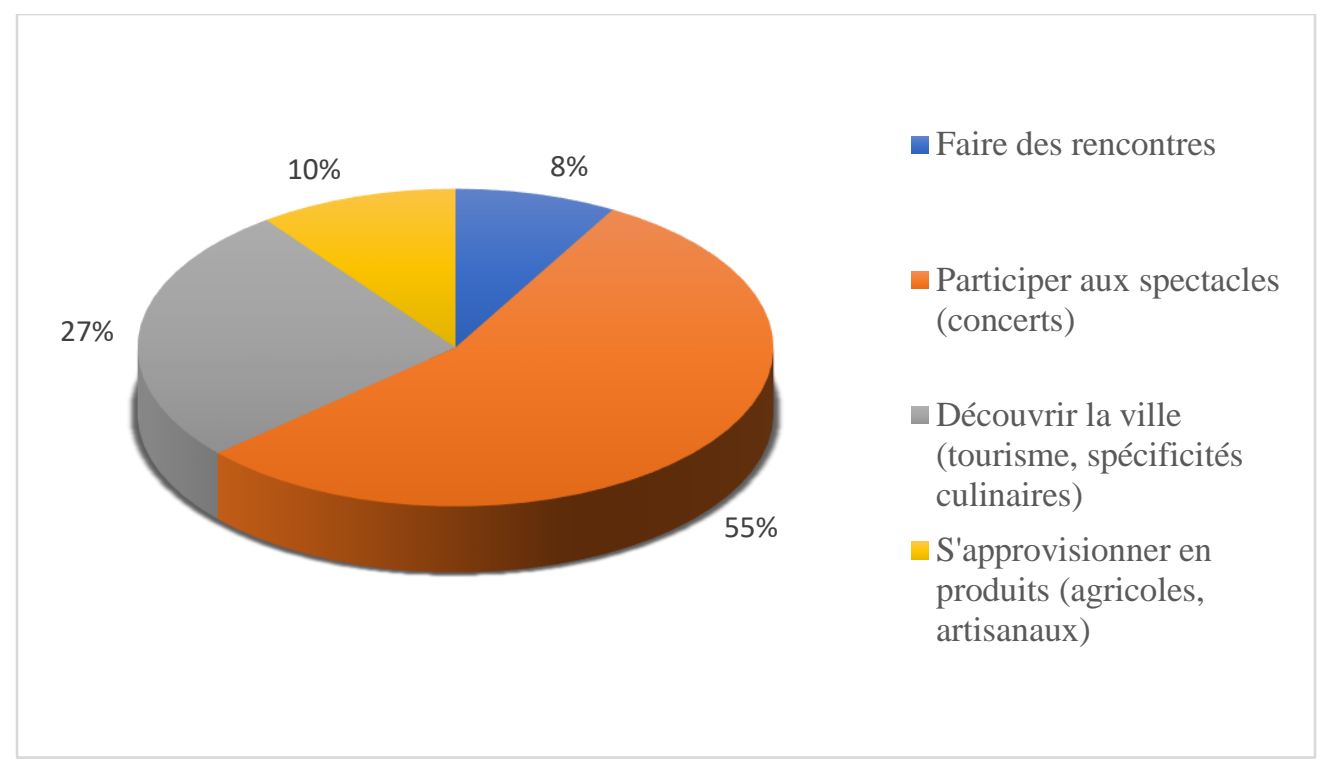

Figure 3 : Motivations des visiteurs de la ville de Savalou, à l'occasion de la fête du 15 août Source : Résultats d'enquêtes, août 2021

Il ressort de cette figure que la participation aux spectacles est la principale raison qui motive les visiteurs $(55 \%)$ de la ville de Savalou, à l'occasion de la fête du 15 août. Elle est suivie de la découverte de la ville (27\%) à travers ses traits physiques et ses spécificités culinaires (en particulier les mets faits à base de l'igname). L'approvisionnement en produits agricoles et artisanaux $(10 \%)$ et les rencontres (8\%) constituent respectivement la troisième et quatrième raison qui motive les visiteurs de la ville. L'aspect festif prend donc le pas sur les autres aspects de l'évènement.

Les spectacles organisés à l'occasion de la fête du 15 août sont les concerts de musique traditionnelle et ceux de musique moderne, le match de football (finale de la coupe de la commune) et l'élection de Miss (la plus belle fille de la commune de Savalou). Les préférences des visiteurs suivant les tranches d'âge sont présentées dans le tableau I ci-après 
Tableau I : Types de spectacle préférés par les visiteurs suivant leurs tranches d'âge

\begin{tabular}{|l|c|c|c|c|}
\hline \multirow{2}{*}{$\begin{array}{l}\text { Tranches } \\
\text { d'âge }\end{array}$} & $\begin{array}{c}\text { Concert de musique } \\
\text { traditionnelle }\end{array}$ & $\begin{array}{c}\text { Concert de } \\
\text { musique moderne }\end{array}$ & Match de football & $\begin{array}{c}\text { Election de Miss } \\
\text { Savalou }\end{array}$ \\
\hline $15-25$ ans & + & ++ & + & ++ \\
\hline $25-35$ ans & + & ++ & + & ++ \\
\hline $35-45$ ans & + & ++ & + & ++ \\
\hline $45-55$ ans & ++ & + & + & + \\
\hline $55-65$ ans & ++ & + & + & + \\
\hline $65-75$ ans & ++ & + & + & + \\
\hline $\begin{array}{l}75 \text { ans et } \\
\text { plus }\end{array}$ & + & + & + & + \\
\hline
\end{tabular}

Source : Résultats d'enquêtes, août 2021

Légende : + Préfère ++ Préfère plus

D'après ce tableau, les visiteurs aiment tous les types de spectacles proposés lors de la fête de 15 août. Mais suivant les âges, on note que les visiteurs de 15 à 45 ans préfèrent plus les concerts de musique moderne et l'élection de Miss Savalou. Quant aux visiteurs âgés de 45 à 75 ans, ils préfèrent plus les concerts de musique traditionnelle. Enfin, les visiteurs de plus de 75 ans aiment indifféremment l'un ou l'autre type de spectacle (il n'y a de préférence particulière à leur niveau).

En combinant les résultats de la figure 3 et ceux du tableau I, on peut affirmer que les spectacles constituent le principal centre d'intérêt des visiteurs de la ville de Savalou à l'occasion de la fête du 15 août et qu'ils préfèrent particulièrement les concerts (musique traditionnelle et musique moderne) et l'élection de Miss. Ce sont informations dont les acteurs communaux gagneraient à tenir compte dans l'organisation de cette fête.

\subsection{Défis de l'inscription territoriale de la fête du 15 août}

Le premier élément sur lequel il importe de mettre l'accent ici est le manque de coordination des manifestations de la fête. Certes, depuis les années 1990, un programme est souvent élaboré à la veille de la fête. Mais dans la pratique, il n'est jamais respecté. La mairie qui devrait jouer le rôle de lead, de coordonnateur n'a jamais eu les capacités de le faire. Il s'en suit un cafouillage dans le déroulement des manifestations qui se traduit par des chevauchements de spectacles, des annulations de spectacles à la dernière minute sans que les participants ne soient informés des raisons de cette annulation, des prestations d'artistes pourtant de renom, qui se déroulent presque dans l'anonymat (sans grand monde parce que la communication autour du spectacle a été mal assurée), etc. 
Le deuxième élément qui est une conséquence du premier est la faible retombée financière de la fête profitant à la mairie. A la date d'aujourd'hui, et se fondant sur les échanges effectués avec les acteurs sur le terrain au cours des éditions de 2019 et de 2021, les montants prélevés au titre de taxes et autres redevances sont insignifiants. Par exemple, les commerçants qui s'installent le long des voies urbaines payeraient 100 à 300FCFA selon la taille de leur étalage. Et aucune statistique fiable n'est disponible sur le nombre de ces commerçants. Deux sociétés de téléphonie mobile (MTN et Moov) payent des droits de publicité à hauteur de 100 000FCFA par opérateur. Quant aux forains, leurs droits de place (5000 FCFA par forain) et autres frais de participation (en moyenne 2000FCFA par forain) sont payés à la structure organisatrice de la foire qui n'en fait aucun compte rendu à la mairie. La fête du 15 août profite donc plus aux acteurs économiques qu'aux finances de la commune.

En l'étape actuelle de l'organisation de cette fête et au vu des constats effectués, il y a lieu de réorganiser l'évènement afin d'assurer son ancrage institutionnel et d'accroitre sa contribution au développement local. Dans cette optique, la mairie peut mettre en place une structure qui s'occupera de la fête sous le format d'une régie autonome ou d'une régie personnalisée. Il s'agit de modes de gestion assurés par les collectivités locales à travers lesquels l'autorité est représentée par une structure qui jouit de l'autonomie financière sans personnalité morale ni juridique (régie autonome) ou avec personnalité morale propre (régie personnalisée). Dans le premier cas (régie autonome), il sera mis en place un conseil d'exploitation (dont les membres seront nommés par le conseil communal) et dans le second cas, on mettra en place un conseil d'administration dont les membres seront également nommés par le conseil communal. Dans l'un ou l'autre cas, l'autorité communale doit s'affirmer par la prise en mains et la coordination de l'ensemble des manifestations entrant dans le cadre de cette fête.

\section{Discussions}

Les observations faites au sujet de la fête de 15 août rejoignent celles de Di Méo (2001) qui explique que les événements urbains s'incrustent dans des lieux permettant des échanges intenses et faciles, des lieux non extensibles car chargés d'un sens et d'une puissance symbolique. Pour Gibout (2000), les évènements urbains qui marquent le plus l'esprit des visiteurs sont les festivals en ce sens qu'ils sont reliés à un site historique ou architectural.

A partir d'une réflexion sur deux évènements organisés à Gênes (Nordouest de l'Italie) et à Lille (Nord de la France), en l'occurrence, l'année «Capitale Européenne de la Culture » en 2004, Gavari-Barbas et Jacquot (2007) indiquent que l'événement urbain est un outil de gouvernance territoriale, ce qui n'est pas le cas pour la ville de Savalou, et qu'en fait, le 
temps festif ne constitue qu'une des dimensions temporelles de l'événement urbain, qui s'appuie sur l'attente, l'urgence, la volonté de durer et la mise en récit pour légitimer les actions menées.

L'ensemble des acteurs interrogés reconnaissent l'importance de la fête du 15 août dans la promotion de la destination Savalou et pensent également qu'elle pourrait offrir un creuset pour l'aménagement de la ville. Mais telle qu'elle s'organise actuellement, cette fête est loin d'impacter durablement l'espace urbain. En effet, les manifestations ne durent véritablement que l'instant d'une journée ou deux au maximum. Déjà le 15 août au soir, beaucoup de forains plient bagages et rares sont les visiteurs urbains qui passent la nuit du 15 au 16 août à Savalou sauf si le 15 août est un vendredi. Ce constat rejoint celui de Augustin et Montané (2004) qui affirment, au sujet des évènements organisés à Toulouse (Sud-Ouest de la France) et à Bordeaux (Sud-Ouest de la France), qu'une fois la fête terminée, les tentes pliées, le palais des congrès libéré, les villes sont laissées à leur rythme habituel sans qu'aucune disposition ne soit prise pour inscrire durablement l'évènement dans l'espace urbain et améliorer sa contribution au développement du territoire. Seuls, le dossier de presse et les souvenirs des participants peuvent témoigner de l'intensité des évènements dès lors qu'ils se terminent.

En réalité, si les évènements urbains étaient bien organisés, ils devraient agir comme des catalyseurs des transformations urbaines à la fois matérielles et immatérielles car, même achevés, ces évènements favorisent la constitution d'une identité collective au sein des villes.

D'après Boogarts (1993), les évènements urbains prennent l'espace de la ville comme support et conduisent à la formation de micro-lieux en termes de rassemblement, d'installations temporaires ou pérennes et d'une reconversion temporaire des usages des lieux qu'ils submergent. L'événement urbain participe donc, de manière éphémère, à la production de lieux dans la mesure où il constitue une forme d'animation sociale et urbaine des espaces dont il se saisit.

Pour Manuel (2012), le portage de l'événement urbain résulte souvent d'un consensus entre certains acteurs clefs de l'évènement. Ceci détermine une nouvelle forme de gouvernance. Pour l'auteur, le leadership de l'autorité communale est déterminant dans le portage institutionnel de l'évènement urbain.

\section{Conclusion et perspectives}

La fête du 15 août est un évènement qui mobilise l'ensemble des acteurs urbains sans pour autant contribuer au développement local. Elle se présente aujourd'hui comme une simple réjouissance populaire plutôt qu'un levier ou un catalyseur des énergies au profit du développement local. $\mathrm{Vu}$ 
l'engouement que suscite cette fête au plan local voire national, il est impérieux que les acteurs impliqués coordonnent davantage leurs efforts, sous le leadership des autorités communales, afin d'accrôtire sa conribution au développement du territoire communal.

\section{References:}

1. Augustin J.- P. et Montané M.-A. (2004): «Différenciation et dualisation de l'action publique : le cas des quartiers fragiles et de la jeunesse urbaine en France ». Lien social et politiques, $\mathrm{n}^{\circ}$ 52, pp. 8193

2. Appert M. (2012) : «Les JO 2012 à Londres : un grand événement alibi du renouvellement urbain à l'est de la capitale », Géo confluences, $\mathrm{n}^{\circ}$, pp. 26-40.

3. Boogarts, I. (1993) : «Festivalomania », Les Annales de la Recherche Urbaine, $\mathrm{n}^{\circ} 57-58$, Espaces publics en ville, pp. 114-119.

4. Di Méo G. (2005) : «Le renouvellement des fêtes et des festivals, ses implications géographiques », Annales de géographie, $\mathrm{n}^{\circ}$ 643, pp. 227 243.

5. Gibout C. (2000): «Transactions dans la ville récréative contemporaine Les espaces publics comme médiateurs sociaux ». Revue Sociologies Dossiers, ${ }^{\circ} 7$, pp 13-32.

6. Gras P. (2007) : "Cardiff veut lier développement et grands événements », Géo carrefour, n82/3, pp. 18-32.

7. Gravari-Barbas M. (2009) : «La ville festive ou construire la ville contemporaine par l'événement». Bulletin de l'Association de géographes français, $\mathrm{n}^{\circ} 3$, pp. 279-290.

8. Gravari-Barbas M. et Jacquot S. (2007) : «L'événement, outil de légitimation de projets urbains : l'instrumentalisation des espaces et des temporalités événementiels à Lille et Gênes », Géo carrefour, $n^{\circ} 82 / 3$, pp. 43-52

9. Gravari-Barbas M. et Veschambre V. (2005) : «S'inscrire dans le temps et s'approprier l'espace : enjeux de pérennisation d'un événement éphémère. Le cas du festival de la $\mathrm{BD}$ à Angoulême ». Les Annales de géographie, pp. 285-306.

10. Gravari-Barbas M. (2000) : La ville festive. Espaces, expressions, acteurs. Thèse pour l'Habilitation à Diriger une Recherche, Université d'Angers, $322 \mathrm{p}$.

11. Mairie de Savalou (2009) : Stratégie de développement touristique de la commune de Savalou. Rapport d'étude, 68p

12. Moiroux F. (2010) : "L'événement, vitrine ou laboratoire de la ville ?», Revue D'architectures, n¹93, pp. 35-49 
13. Nassourou I. et Sani M. M. (2019) : « La guerre de 2011 en Libye et les migrants de retour dans la Région de Tahoua Au Niger : Entre incertitude sécuritaire et défis de développement local ». European Scientific Journal, vol.15, No.35, pp. 32-48.

14. Nkwenka Nyanda P. G., Temomo Wamba R. et Mimché Kouotou A. C. (2020) : «Décentralisation fiscale et croissance des dépenses locales dans les collectivités territoriales décentralisées au Cameroun : cas de la ville de Douala ». European Scientific Journal. Vol.16, No.4, pp. 74-88. 